\title{
Erratum
}

\section{A CNDO CI Study of the Electronic Structure and Spectrum of Nitrobenzene}

\author{
B. TINLAND
}

Theoret. chim. Acta (Berl.) 13, 171-174 (1969)

Received January 12, 1970

An error was found in the atomic coordinates and new computations were performed. Tables 2, 3 and 4 and some results should be corrected as follows:

Table 2. Atomic coordinates $(\AA)$

\begin{tabular}{lll}
\hline Atom & $x$ & $y$ \\
\hline $\mathrm{O}_{1}$ & -1.03718 & 0. \\
$\mathrm{~N}$ & 0. & 0.6232 \\
$\mathrm{C}_{1}$ & 0. & 2.1132 \\
$\mathrm{C}_{2}$ & -1.22068 & 2.73516 \\
$\mathrm{C}_{3}$ & -1.19573 & 4.16495 \\
$\mathrm{C}_{4}$ & 0. & 4.83464 \\
$\mathrm{H}_{1}$ & -2.14643 & 2.07072 \\
$\mathrm{H}_{2}$ & -2.12629 & 4.72309 \\
$\mathrm{H}_{3}$ & 0. & 5.91464 \\
\hline
\end{tabular}

Table 3. Singlet transitions

\begin{tabular}{cc}
\hline This work \\
\hline $3.27(0)$. \\
$3.83(0)$. \\
$x \quad 4.68(0.002)$ \\
$y$ & $5.12(0.002)$ \\
$x$ & $5.84(0.03)$ \\
$y$ & $6.67(0.58)$ \\
\hline
\end{tabular}

Table 4. Valence electrons populations

\begin{tabular}{lccccccccc}
\hline & $\mathrm{O}_{1}$ & $\mathrm{~N}$ & $\mathrm{C}_{1}$ & $\mathrm{C}_{2}$ & $\mathrm{C}_{3}$ & $\mathrm{C}_{4}$ & $\mathrm{H}_{1}$ & $\mathrm{H}_{2}$ & $\mathrm{H}_{3}$ \\
\hline $1 s$ & & & & & & & 0.94 & 0.97 & 0.97 \\
$2 s$ & 1.74 & 1.14 & 1.02 & 1.05 & 1.03 & 1.02 & & & \\
$2 p_{x}$ & 1.43 & 1.03 & 1.02 & 1.00 & 1.00 & 1.00 & & & \\
$2 p_{y}$ & 1.78 & 1.11 & 0.84 & 0.99 & 0.99 & 1.00 & & & \\
$2 p_{z}$ & 1.52 & 0.98 & 1.07 & 0.98 & 0.99 & 0.97 & & & \\
\hline Total & 6.47 & 4.26 & 3.95 & 4.02 & 4.01 & 3.99 & & & \\
\hline
\end{tabular}


164 B. Tinland: A CNDO CI Study of the Electronic Structure and Spectrum of Nitrobenzene

The computed values for the dipole moment and the ionization potential are now 5.27 Debyes and $11.43 \mathrm{eV}$, respectively.

I wish to thank Prof. E. Heilbronner and Dr. H. Baumann for bringing this etror to my attention.

Dr. B. Tinland

Université de Lyon

Section de Recherches de Mécanique

Ondulatoire Appliquée

43 Boulevard de l'Hippodrome

F-69 Villeurbanne 